\title{
The role of velocity-neutral creep on the modulation of tectonic tremor activity by periodic loading
}

\author{
Thomas J. Ader, ${ }^{1,2}$ Jean-Paul Ampuero, ${ }^{1}$ and Jean-Philippe Avouac ${ }^{1}$ \\ Received 9 May 2012; revised 27 July 2012; accepted 1 August 2012; published 31 August 2012.
}

[1] Slow slip events and associated non-volcanic tremors are sensitive to oscillatory stress perturbations, such as those induced by tides or seismic surface waves. Slow slip events and tremors are thought to occur near the seismic-aseismic transition regions of active faults, where the difference $a-b$ $=\partial \mu / \partial \ln V$ between the sensitivity of friction to slip rate and fault state, which characterizes the stability of slip, can be arbitrarily small. We investigate the response of a velocitystrengthening fault region to oscillatory loads through analytical approximations and spring-slider simulations. We find that fault areas that are near velocity-neutral at steadystate, i.e., $\partial \mu / \partial \ln V \approx 0$, are highly sensitive to cyclic loads: oscillatory stress perturbations in a certain range of periods induce large transient slip velocities. These aseismic transients can in turn trigger tremor activity with enhanced oscillatory modulation. In this sensitive regime, a harmonic Coulomb stress perturbation of amplitude $\Delta S$ causes a slip rate perturbation varying as $e^{\Delta S(a-b) \sigma}$, where $\sigma$ is the effective normal stress. This result is in agreement with observations of the relationship between tremor rate and amplitude of stress perturbations for tremors triggered by passing seismic waves. Our model of tremor modulation mediated by transient creep does not require extremely high pore fluid pressure and provides a framework to interpret the sensitivity and phase of tidally modulated tremors observed in Parkfield and Shikoku in terms of spatial variations of friction parameters and background slip rate. Citation: Ader, T. J., J.-P. Ampuero, and J.-P. Avouac (2012), The role of velocity-neutral creep on the modulation of tectonic tremor activity by periodic loading, Geophys. Res. Lett., 39, L16310, doi:10.1029/2012GL052326.

\section{Introduction}

[2] The recent discovery of slow-slip events (SSEs) and non-volcanic tremors (NVTs) has lead to a vast body of observational work in the past decade. SSEs and NVTs appear to coincide in time and space [e.g., Rogers and Dragert, 2003] and have been observed in various tectonic settings [Schwartz and Rokosky, 2007; Brown et al., 2009; Shelly et al., 2011]. Rubinstein et al. [2008], Nakata et al. [2008] and Thomas et al. [2009, 2012] reported a modula-

\footnotetext{
${ }^{1}$ Department of Geological and Planetary Sciences, California Institute of Technology, Pasadena, California, USA.

${ }^{2}$ Laboratoire de Géologie, Ecole Normale Supérieure, CNRS, Paris, France.

Corresponding author: T. J. Ader, Department of Geological and Planetary Sciences, California Institute of Technology, Pasadena, CA 91125, USA. (ader@caltech.edu)

C2012. American Geophysical Union. All Rights Reserved. 0094-8276/12/2012GL052326
}

tion of NVT rates by tidal stresses of a few $\mathrm{kPa}$ or less, in the Cascadia subduction zone, in southwest Japan and on the deep San Andreas Fault at Parkfield, respectively. Hawthorne and Rubin [2010] inferred a modulation of the slip rate of SSEs by tidal stresses studying borehole strainmeter data in Cascadia. Miyazawa and Brodsky [2008] found that NVTs in western Japan were triggered by the passing surface waves radiated by the 2004 Sumatra earthquake, and observed an exponential relationship between the amplitude of the NVTs and the Coulomb stress perturbation in the source region.

[3] Nakata et al. [2008] and Thomas et al. [2009, 2012] explained the correlation of NVTs with tidal loading with the model of Dieterich [1994], therefore postulating that tidal stresses directly triggered seismic slip on locked asperities. This interpretation required very low values of $a \sigma$, either implying $a$ orders of magnitude lower than values inferred from lab experiments [Blanpied et al., 1995] or extremely low effective normal stresses. They retained the second hypothesis (Nakata et al. [2008] proposed $\sigma_{\text {eff }} \approx 100 \mathrm{kPa}$ while Thomas et al. [2009] found $\sigma_{\text {eff }} \approx 9$ to $35 \mathrm{kPa}$ ), and justified it by a nearly lithostatic pore pressure. However, since the nucleation size on an asperity is inversely proportional to the effective normal stress [e.g., Rubin and Ampuero, 2005], this would imply large nucleation sizes for seismic ruptures, in contradiction with the prevailing view that tremors are small shear rupture events. Miyazawa and Brodsky [2008] explained the exponential relationship between tremor amplitude variations and amplitude of the incoming waves with a pre-existing exponential distribution of failure stresses within the tremor source region.

[4] Velocity-weakening fault patches can show enhanced sensitivity to oscillatory loads, but only over a narrow range of patch sizes and loading periods [Perfettini and Schmittbuhl, 2001; Lowry, 2006].

[5] Here we present an alternative mechanism for these observed correlations, relying on the fact that tremors usually happen at the transition between the rate-strengthening and rate-weakening parts of a fault, thus a region where the sensitivity of steady-state friction to velocity, $a-b=\partial \mu /$ $\partial \ln V$, can be arbitrarily low. We first present the response of a spring-slider system with rate-strengthening rheology to harmonic shear and normal stress perturbations of different periods. We then establish a non-linear, exponential relationship between the amplitudes of the slip rate and the stress perturbations for large enough Coulomb stress perturbations.

\section{Model Hypotheses}

[6] We adopt the view proposed by Ide et al. [2007] and Shelly et al. [2011] that tremors are generated by the rupture of small rate-weakening asperities caused by slip on the 

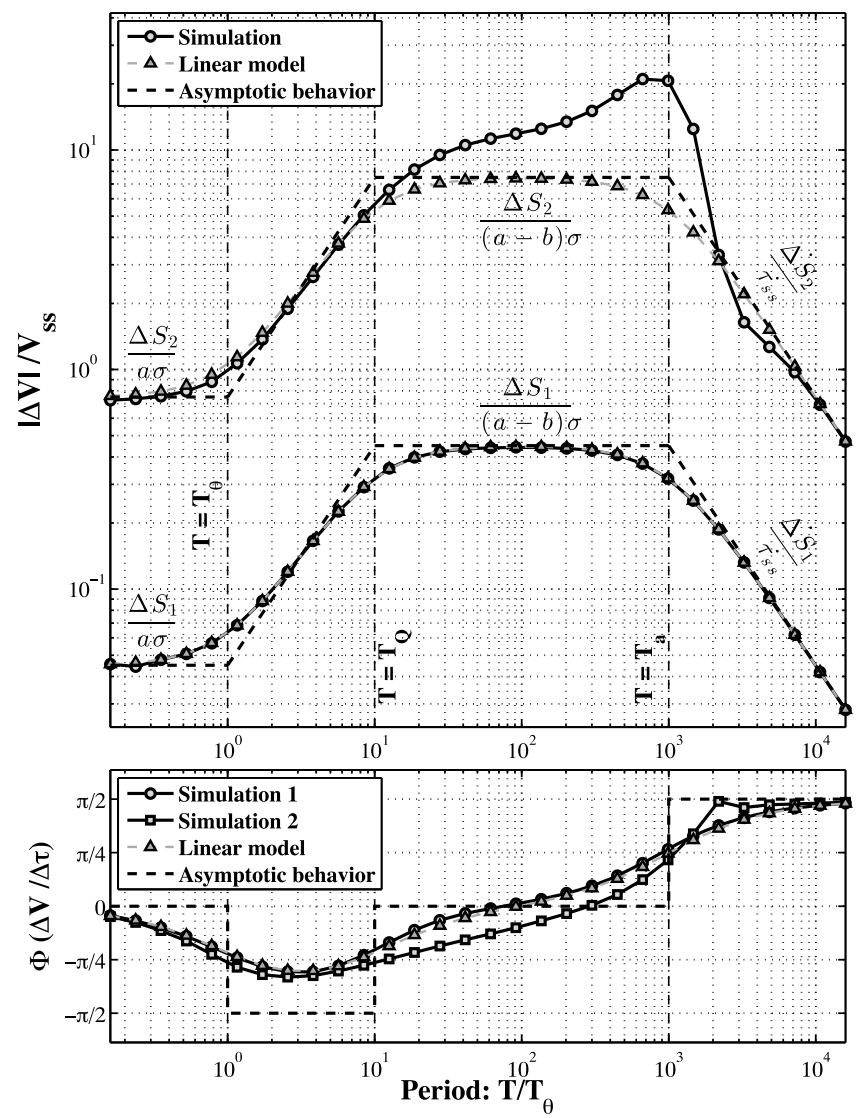

Figure 1. Response of a spring-slider system to small harmonic Coulomb stress perturbations of different periods and amplitudes $\Delta S_{1}=0.9 \mathrm{kPa}$ (simulation 1: $\Delta \tau_{1}=\Delta \sigma_{1}=$ $3 \mathrm{kPa}$ ) and $\Delta S_{2}=15 \mathrm{kPa}$ (simulation 2: $\Delta \tau_{2}=\Delta \sigma_{2}=$ $50 \mathrm{kPa}$ ). The system is undergoing constant loading at velocity $V_{\mathrm{ss}}=0.02 \mathrm{~m} / \mathrm{yr}$ under mean normal stress $\sigma_{o}=5 \mathrm{MPa}$. The normalized spring stiffness is $k / \sigma_{o}=0.002 \mathrm{~m}^{-1}$. The other parameters are: $\mu_{\mathrm{ss}}=0.7, a=0.004, b=0.0036$ and $D_{c}=$ $0.2 \mathrm{~mm}$. (top) Amplitude of the creep rate variations. The black lines with circles represent the results of the simulations (one circle for each period tested). The dashed grey lines with triangles represent the small perturbation approximation (equation (3)) for each simulation while the dashed black lines indicate the corresponding asymptotic behavior of the system with equations indicated on the plot. The critical periods $T_{\theta}, T_{Q}$ and $T_{a}$ are also indicated on the plot. (bottom) Phase difference between the creep rate and the Coulomb stress variations.

surrounding plate interface. Under the assumption that the rupture is Coulombian, the NVT rate is directly proportional to the slip rate on the fault.

[7] We thus study the response of a rate-strengthening fault to a stress perturbation, modeling the fault as a onedimensional spring-slider system with stiffness $k$ [e.g., Perfettini et al., 2001; Parsons, 2005], loaded at constant background velocity $V_{\mathrm{ss}}$, under shear stress perturbation $\Delta \tau(t)=\Delta \tau e^{i \omega t}$ and perturbed normal stress $\sigma(t)=\sigma_{o}+\Delta \sigma e^{i \omega t}$, where the amplitude of the perturbation is smaller than the prevailing normal stress $\left(\Delta \sigma<\sigma_{o}\right)$. Both shear and normal stress perturbations are supposed to be in phase for the sake of simplicity. The evolution of the friction coefficient $\mu$ is described by a rate-and-state law [e.g., Marone, 1998]:

$$
\mu=\mu_{\mathrm{ss}}+a \ln \frac{V}{V_{\mathrm{ss}}}+b \ln \frac{\theta V_{\mathrm{ss}}}{D_{c}},
$$

where $V$ is the total slip rate of the slider, $\theta$ a fault state variable, $\mu_{\mathrm{ss}}$ the steady-state friction coefficient at slip rate $V_{\mathrm{ss}}$, $D_{c}$ the characteristic slip for friction to evolve between two steady states, and $a$ and $b$ are constitutive fault parameters verifying $a-b>0$, such that the system has a ratestrengthening rheology. The state variable $\theta$ evolves according to the "aging law" [e.g., Marone, 1998]:

$$
\frac{d \theta}{d t}=1-\frac{V \theta}{D_{c}} .
$$

\section{Period Dependent Response of the System}

[8] When the amplitude of the harmonic perturbations of Coulomb stress $\Delta S=\Delta \tau-\mu_{\mathrm{ss}} \Delta \sigma$ is small enough (i.e., $\Delta S \ll(a-b) \sigma)$, the slip rate of the slider undergoes small harmonic variations around its steady state value: $V(t)=V_{\mathrm{ss}}+\Delta V e^{i \omega t}$, where $\Delta V \ll V_{\mathrm{ss}}$. The resulting perturbations of slip rate $\Delta V$ can be obtained by a linearized approximation [Segall, 2010]:

$$
\frac{\Delta V}{V_{\mathrm{ss}}}=\frac{i \omega}{1+i \omega t_{a}(\omega)} \frac{\Delta S}{\dot{\tau}_{s s}},
$$

where $\omega=2 \pi / T$ is the pulsation, $\dot{\tau}_{s s}=k V_{\mathrm{ss}}$ the background stressing rate, $t_{a}(\omega)=A(\omega) \sigma / \tau_{\mathrm{ss}}$, and

$$
A(\omega)=a-\frac{b}{1+i \omega \theta_{\mathrm{ss}}}
$$

is a period dependent constitutive fault parameter. In the limits $\omega \theta_{\mathrm{ss}} \ll 1$ and $\omega \theta_{\mathrm{ss}} \gg 1, A$ becomes real $(A=a-b$ and $A=a$, respectively) and quantifies the velocity dependence of the friction in the steady-state regime.

[9] Equation (3) is represented for two different values of $\Delta S$ in Figure 1 (dashed line with triangles), such that $\Delta S_{1}<(a-b) \sigma$ and $\Delta S_{2}>(a-b) \sigma$. Three characteristic periods bounding different behaviors of the system appear. $T_{\theta}=2 \pi \theta_{\mathrm{ss}}=2 \pi D_{c} / V_{\mathrm{ss}}$, where $\theta_{\mathrm{ss}}$ is the steady-state value of the state variable, defines the characteristic time scale for the evolution of the state variable. For perturbations with period $T<T_{\theta}$, the state variable does not have time to evolve and the rate-and-state law reduces to a purely rate-dependent law with $\partial \mu / \partial \ln V=a . T_{Q}=T_{\theta} \times a /(a-b)>T_{\theta}$ is the period above which the state variable has time to fully adjust so that in the steady state, $\partial \mu / \partial \ln V=a-b$. The third period, $T_{a}$, is the one for which $\left|\omega t_{a}(\omega)\right|=1$ and separates between two physically different responses of the system to the stress perturbation. For periods $T>T_{a}$, the damping due to the friction acts on a much smaller time-scale than that of the characteristic evolution of the spring-slider and the response of the system becomes that of a perturbed spring-slider in steady state with no friction. In this quasi-static regime, equation (3) reduces to:

$$
\frac{\Delta V}{V_{\mathrm{ss}}}=\frac{\Delta \dot{S}}{\dot{\tau}_{s s}} .
$$



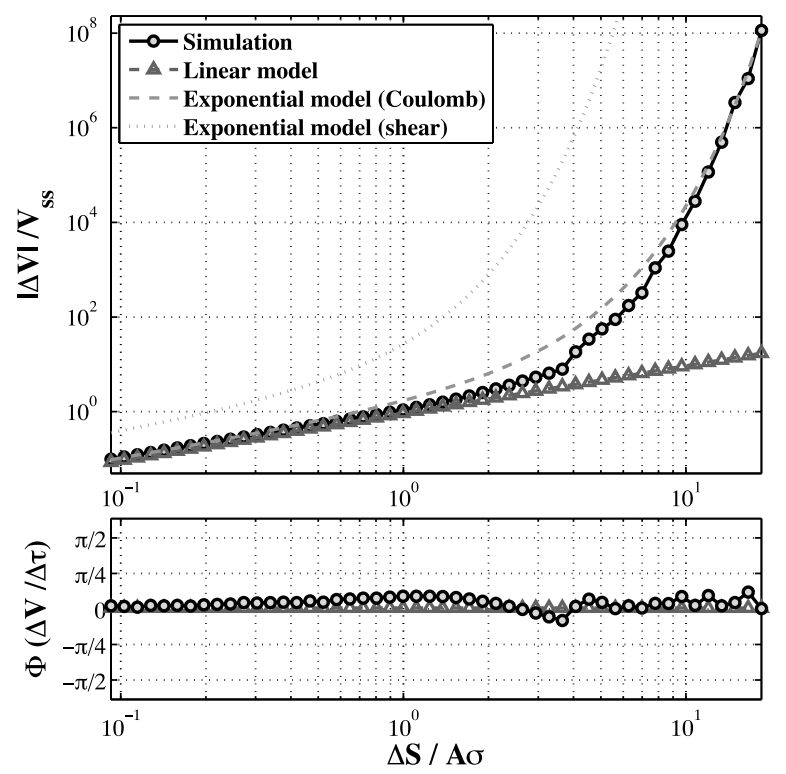

Figure 2. Non linear response of a spring-slider system to small harmonic stress perturbations for different amplitudes. The period $T$ of the perturbation is such that $T / T_{Q}=T_{a} / T=$ 2.5. The parameters are the same as in Figure 1, except for the fault parameter $b=0.00385$ and $D_{c}=0.5 \mathrm{~mm}$, so that $A=|A(\omega)|=1.08(a-b) \approx(a-b)$. The meaning of the different lines is given in the legend. (top) Amplitude of the creep rate variations. The exponential models derive from equation (9) taking either the Coulomb stress or only the shear stress, and replacing $(a-b)$ by the actual value of $A$. The linear model corresponds to equation (3). (bottom) The phase difference between the creep rate and the Coulomb stress variations.

For $T<T_{a}$, the period of the velocity oscillations is too small for the spring stiffness to have any significant effect, and the system evolves as a simple slider with a rate-and-state friction law. In this regime, the amplitude of the velocity perturbation is proportional to the amplitude of the stress perturbation:

$$
\frac{\Delta V}{V_{\mathrm{ss}}}=\frac{\Delta S}{A \sigma}
$$

where $A=|A(\omega)|$ depends on the period. In particular, when $T_{Q}<T, A \approx(a-b)$ and so the amplitude of the velocity oscillations becomes:

$$
\frac{\Delta V}{V_{\mathrm{ss}}}=\frac{\Delta S}{(a-b) \sigma}
$$

which may result in large oscillations of the slip rate for small values of $(a-b)$.

[10] The ratio $T_{a} / T_{\theta}$ has the following expression:

$$
\left(\frac{T_{a}}{T_{\theta}}\right)^{2}=\frac{1}{2}\left[\sqrt{4 \tilde{a}^{2}+\left(1-[\tilde{a}-\tilde{b}]^{2}\right)^{2}}-\left(1-[\tilde{a}-\tilde{b}]^{2}\right)\right]
$$

where $\tilde{a}=a \sigma / k D_{c}$ and $\tilde{b}=b \sigma / k D_{c}$. A graphical representation of equation (8) is given in Figure S1 in the auxiliary material. ${ }^{1} \mathrm{~A}$ response following equation (7) requires $T_{a}$ / $T_{\theta} \gg 1$, which is possible only when $\tilde{a}-\tilde{b} \gg 1$, in which case $T_{a} / T_{\theta}=\tilde{a}-\tilde{b}$.

[11] In order to assess the validity of the linear approximation, we simulate the general response of a spring-slider system to harmonic shear and normal stress perturbations of equal amplitudes, solving the equations of motion using a Runge-Kutta algorithm with a fifth-order adaptive step-size control [Press et al., 1992] for Coulomb stress perturbations of amplitudes $\Delta S_{1}$ and $\Delta S_{2}$. The results are plotted on Figure 1 and show that in the first case, the linear approximation is justified, while in the second case, for periods $T_{Q}<$ $T<T_{a}$, the amplitude of the slip perturbation becomes non linear and greater than what equation (3) predicts.

\section{Influence of Coulomb Stress Amplitude}

[12] Over the range of periods $T_{Q}<T<T_{a}$, Coulomb stress perturbations of amplitude greater than $(a-b) \sigma$ induce non linear velocity fluctuations of large amplitude. In Figure $1, T_{a} / T_{Q}=(a-b) / a \times T_{a} / T_{\theta}=10^{2}$, but can actually be several orders of magnitude larger for a different set of parameters values. For instance, $D_{c}=2 \mu \mathrm{m}$ [e.g., Marone, 1998] increases this ratio to $10^{4}$. This non-linear amplification of the response can thus prevail over a range of periods spanning several orders of magnitude.

[13] To first approximation, the induced non linear velocity fluctuations depend exponentially on the stress perturbation (see derivation in Appendix A):

$$
\frac{\Delta V}{V_{\mathrm{ss}}} \approx e^{\frac{\Delta S}{(a-b) \sigma}} .
$$

Equation (7) is simply a linear approximation of equation (9) when $\Delta S \ll(a-b) \sigma$. Figure 2 shows the result of a simulation with shear and normal stress perturbations of period $T_{a} / T_{Q}=T_{a} / T>1$, and increasing amplitudes. This simulation shows that the exponential approximation (equation (9)) provides a good description of the system's behavior. This statement still holds when considering the "slip law" [e.g., Marone, 1998] instead of the "aging law" for the evolution of the state variable (Figure S2).

[14] This simulation also predicts a correlation of the slip perturbation with the Coulomb stress rather than with the shear stress perturbation. This point has been discussed in the observational literature, but unfortunately the conclusions remain elusive and thus hard to compare with our model predictions. Both Nakata et al. [2008] and Miyazawa and Brodsky [2008] reported a correlation of tremors with the Coulomb stress perturbations, while Thomas et al. [2009, 2012] and Hawthorne and Rubin [2010] observed a correlation with shear stress variations only. However, Thomas et al. [2009, 2012] found the best correlation for an extremely small friction coefficient $(\mu=0.02)$, while Hawthorne and Rubin [2010] noted that if fluids did not diffuse significantly over the time scale of tides, the changes in pore pressure could compensate the applied normal stress variations, resulting in very small effective normal stress

${ }^{1}$ Auxiliary materials are available in the HTML. doi:10.1029/ 2012 GL052326. 
variations. In both cases, the effective Coulomb stress and shear stress variations were almost the same, making it impossible to ascertain whether tremors correlated better with the one or the other.

[15] Looking at the phase difference $\Phi(\Delta V / \Delta \tau)$ between the slip rate and the stress variations (Figure 2, bottom) indicates that, at periods for which the sensitivity is the highest, NVTs should correlate with stress perturbations rather than with perturbations of the stress rate $(\Phi=0$ and not $\pi / 2)$. We will come back to this point in the discussion.

\section{Discussion and Conclusions}

[16] We here propose a mechanism to explain the observed correlation of NVTs with tidal stresses and passing seismic surface waves [Miyazawa and Brodsky, 2008; Nakata et al., 2008], as well as the apparent tidal modulation of slow slip in Cascadia [Hawthorne and Rubin, 2010]. The idea relies on the fact that both NVTs and SSEs seem to occur right below the locked section of faults, where the fault constitutive parameters define a nearly velocity-neutral zone $(a-b \approx 0)$. We show that for a certain range of periods, a harmonic perturbation of the Coulomb stress on such a fault can induce a large perturbation of the slip rate around its steady-state value, of amplitude varying exponentially with the amplitude of the Coulomb stress perturbation. Assuming that NVTs are due to the rupture of rate-weakening $(a-b<0)$ patches embedded in that fault region, the tremor rate should be proportional to the transient aseismic slip velocity. This can explain the sensitivity of NVTs to tidal and seismic stresses without requiring unusual values for $a$ and $b$ nor requiring extremely low effective normal stresses. This also provides an alternative explanation to the exponential relationship between NVTs and passing surface wave amplitudes without resorting to ad-hoc exponential distributions of initial stresses [Miyazawa and Brodsky, 2008].

[17] This model predicts a correlation of NVTs with varying stresses only for a bounded range of periods, $T_{Q}<$ $T<T_{a}$, which can vary in space and time yielding inhomogeneities of the sensitivity of NVTs to stress perturbations. In Parkfield, Thomas et al. [2012] observed an increase of sensitivity at tidal periods as a function of depth and closeness to the creeping segment, i.e., towards regions where $V_{\mathrm{ss}}$ is expected to be larger. Given that both $T_{Q}$ and $T_{a}$ are inversely proportional to $V_{\text {ss }}$, those observations can be explained by the fact that as $V_{\mathrm{ss}}$ decreases, $T_{Q}$ increases to values that might become higher than the tidal period, thus inhibiting the correlation. Other parameters, such as $D_{c}, a$ and $b$, might also induce spatial variations of the sensitivity by acting on the bounding periods. Inhomogeneities of $(a-b)$ have two effects: they affect the bounding periods and they directly influence the amplitude of the correlation, according to equation (9). The existence of NVTs itself, and thus of rate-weakening patches $(a-b<0)$ within the creeping zone $(a-b \gtrsim 0)$, may stand as a direct manifestation of the nonuniformity of $(a-b)$ on the fault. This could explain variations of sensitivity to tides in Shikoku [Ide, 2010], which pattern seems too erratic to be explained by local variations of the creep rate. Finally, the analysis of the phase suggests that NVTs should correlate and roughly be in phase with the perturbing stress (Figure 2). However, Figure 1 shows that should the period of the perturbation get closer to $T_{Q}$ or $T_{a}$, the maximum NVT rate would respectively happen slightly before or after the maximum stress perturbation. This could explain why Nakata et al. [2008] observed a time advance of tremors relative to tidal stresses (and concluded of a correlation with the stress rate coupled with a delayed nucleation), while Thomas et al. [2012] reported a slight time lag. In the latter study, if our interpretation of the loss of sensitivity due to decreasing $V_{\text {ss }}$ is correct, the loss of correlation should go hand in hand with an increase of the phase lag.

[18] Stress perturbations due to either tides or passing surface waves have been reported to be of the order of a few kPa [Miyazawa and Brodsky, 2008; Nakata et al., 2008; Thomas et al., 2012]. For an effective normal stress of $300 \mathrm{MPa}$ and stress perturbations of amplitude $3 \mathrm{kPa}$, this exponential regime would be observed for $0<a-b<10^{-5}$. Although such values might appear very small, they may prevail at the transition between the rate-weakening and rate-strengthening parts of the fault, where NVTs and SSEs are observed to originate. For $(a-b) / a=10^{-2}$, $V_{\mathrm{ss}} \sim 1 \mathrm{~m} / \mathrm{yr}$ [e.g., Schwartz and Rokosky, 2007] and $D_{c} \sim 3 \mu \mathrm{m}$ [e.g., Marone, 1998], $T_{Q}$ is of the order of half a day, the dominant period of tides. One needs smaller $D_{c}$ or larger $V_{\mathrm{ss}}$ for $T_{Q}$ to become of the order of the seismic waves period.

[19] In order to apply this mechanism to real faults, small values of $(a-b)=\partial \mu / \partial \ln V$ are necessary over large enough regions, in order to sustain the high sensitivity of tremors to oscillatory stresses. Shimamoto [1986] and Moore et al. [1997] reported a N-shaped dependence of the steady-state friction on $\ln V_{\text {ss }}$ during lab experiments on halite and chyrsotile serpentine respectively, thus unraveling two critical velocities for which $\partial \mu / \partial \ln V=0$. Estrin and Bréchet [1996] and more recently Beeler [2009] proposed models for frictional sliding with a N-shaped curve for the velocity dependence of the friction coefficient. Shibazaki and Iio [2003] and Shibazaki and Shimamoto [2007] subsequently used similar friction laws in simulations of slow-slip events. In those models, the creep velocity during the SSE was such that $\partial \mu /$ $\partial \ln V \approx 0$. The previous spring-slider analysis can then be applied to this configuration, and the spatial extent of the zone with very small values of $a-b$ is as large as the region of active slow slip. This altogether qualitatively reconciles the various observations of correlation of slow slip events and associated non volcanic tremors to stress perturbations induced by tides and passing seismic waves, with fault parameters in agreement with laboratory values, no drastic constraints on local pore pressures, or initial distribution of stresses.

[20] Although the present study focuses on tremors and SSEs, this mechanism might also be applied to regular earthquakes in some situations. In Nepal for instance, Bollinger et al. [2004] reported annual modulations of the seismicity, which turned out to be linked to small stress perturbations of a few $\mathrm{kPa}$, due to varying surface loads caused by the local hydrological cycle [Bettinelli et al., 2008]. Modulation of seismicity by daily tides of similar stress amplitudes was not found. Given that the correlating seismicity forms a belt falling at the transition between the locked and creeping zones of the fault [Ader et al., 2012], the seasonal variations of the seismicity rate might be related to 
the mechanism proposed here. The lack of sensitivity to daily loadings is explained by our model if the period $T_{Q}$ lies between one day and one year.

\section{Appendix A: Coulomb Stress Perturbation of Large Amplitude}

[21] If $(a-b)$ is very small, the small perturbation hypothesis might not be valid anymore. In the steady-state regime, for periods such that $T_{Q}=a T_{\theta} /(a-b)<T<T_{a}$, the equation of motion of the system becomes:

$$
\Delta \tau e^{i \omega t}=\dot{\tau}_{\mathrm{ss}}\left[\frac{\delta(t)}{V_{\mathrm{ss}}}-t\right]+\left(\sigma_{o}+\Delta \sigma e^{i \omega t}\right)\left[\mu_{\mathrm{ss}}+A \ln \frac{V(t)}{V_{\mathrm{ss}}}\right]
$$

where $A=|A(\omega)| \approx(a-b)$. The two terms in the right-hand side of the equation represent respectively the elastic stress due to the spring and the friction on the slider. Now, if the slider reaches high velocities but over a short time, its overall displacement remains small and the elastic force will have little impact on the system. The friction then dictates the evolution of the slider, and equation (10) reduces to:

$$
\left(\Delta \tau-\mu_{\mathrm{ss}} \Delta \sigma\right)=A \sigma \ln \frac{\Delta V}{V_{\mathrm{ss}}},
$$

which leads to the following relation between the velocity and Coulomb stress perturbations:

$$
\frac{\Delta V}{V_{\mathrm{ss}}}=e^{\frac{\Delta S}{A \sigma}}
$$

[22] Acknowledgments. This research was supported by NSF grants EAR-1015698 and EAR-0838495, the Gordon and Betty Moore Foundation and the Southern California Earthquake Center (funded by NSF Cooperative Agreement EAR-0106924 and USGS Cooperative Agreement EAR-0106924, NSF grant EAR0838495 and USGS Cooperative Agreement 02HQAG0008). We thank Paul Segall and another anonymous reviewer for useful comments. This paper is Caltech Tectonics Observatory contribution 206, Caltech Seismolab contribution 10081 and SCEC contribution 1647.

[23] The Editor thanks Paul Segall and an anonymous reviewer for assisting in the evaluation of this paper.

\section{References}

Ader, T., et al. (2012), Convergence rate across the Nepal Himalaya and interseismic coupling on the Main Himalayan Thrust: Implications for seismic hazard, J. Geophys. Res., 117, B04403, doi:10.1029/ 2011JB009071.

Beeler, N. M. (2009), Constructing constitutive relationships for seismic and aseismic fault slip, Pure Appl. Geophys., 166, 1775-1798.

Bettinelli, P., J.-P. Avouac, M. Flouzat, L. Bollinger, G. Ramillien, S. Rajaure, and S. Sapkota (2008), Seasonal variations of seismicity and geodetic strain in the Himalaya induced by surface hydrology, Earth Planet. Sci. Lett., 266, 332-344.

Blanpied, M. L., D. A. Lockner, and J. D. Byerlee (1995), Frictional slip of granite at hydrothermal conditions, J. Geophys. Res., 100, 13,045-13,064.

Bollinger, L., J. P. Avouac, R. Cattin, and M. R. Pandey (2004), Stress buildup in the Himalaya, J. Geophys. Res., 109, B11405, doi:10.1029/ 2003JB002911.
Brown, J. R., G. C. Beroza, S. Ide, K. Ohta, D. R. Shelly, S. Y. Schwartz, W. Rabbel, M. Thorwart, and H. Kao (2009), Deep low-frequency earthquakes in tremor localize to the plate interface in multiple subduction zones, Geophys. Res. Lett., 36, L19306, doi:10.1029/2009GL040027.

Dieterich, J. (1994), A constitutive law for rate of earthquake production and its application to earthquake clustering, J. Geophys. Res., 99, 2601-2618.

Estrin, Y., and Y. Bréchet (1996), On a model of frictional sliding, Pure Appl. Geophys., 147(4), 745-762.

Hawthorne, J. C., and A. M. Rubin (2010), Tidal modulation of slow slip in Cascadia, J. Geophys. Res., 115, B09406, doi:10.1029/2010JB007502.

Ide, S. (2010), Striations, duration, migration and tidal response in deep tremor, Nature, 466, 356-359.

Ide, S., D. R. Shelly, and G. C. Beroza (2007), Mechanism of deep low frequency earthquakes: Further evidence that deep non-volcanic tremor is generated by shear slip on the plate interface, Geophys. Res. Lett., 34 L03308, doi:10.1029/2006GL028890.

Lowry, A. R. (2006), Resonant slow fault slip in subduction zones forced by climatic load stress, Nature, 442, 802-805.

Marone, C. (1998), Laboratory-derived friction laws and their application to seismic faulting, Annu. Rev. Earth Planet. Sci., 26, 643-696.

Miyazawa, M., and E. E. Brodsky (2008), Deep low-frequency tremor that correlates with passing surface waves, J. Geophys. Res., 113, B01307, doi:10.1029/2006JB004890.

Moore, D. E., D. A. Lockner, M. Shengli, R. Summers, and J. D. Byerlee (1997), Strengths of serpentinite gouges at elevated temperatures, J. Geophys. Res., 102, 14,787-14,801.

Nakata, R., N. Suda, and H. Tsuruoka (2008), Non-volcanic tremor resulting from the combined effect of Earth tides and slow slip events, Nat. Geosci., 1, 676-678, doi:10.1038/ngeo288.

Parsons, T. (2005), A hypothesis for delayed dynamic earthquake triggering, Geophys. Res. Lett., 32, L04302, doi:10.1029/2004GL021811.

Perfettini, H. and J. Schmittbuhl (2001), Periodic loading on a creeping fault: Implications for tides, Geophys. Res. Lett., 28, 435-438.

Perfettini, H., J. Schmittbuhl, J. R. Rice, and M. Cocco (2001), Frictional response induced by time-dependent fluctuations of the normal loading, J. Geophys. Res., 106, 13,455-13,472.

Press, W., S. Teukolsky, W. Vetterling, and B. Flannery (1992), Numerical Recipes in C: The Art of Scientific Computing, Cambridge Univ. Press, Cambridge, U. K.

Rogers, G., and H. Dragert (2003), Episodic tremor and slip on the Cascadia subduction zone: The chatter of silent slip, Science, 300(5627), 1942-1943.

Rubin, A. M., and J.-P. Ampuero (2005), Earthquake nucleation on (aging) rate and state faults, J. Geophys. Res., 110, B11312, doi:10.1029/ 2005JB003686.

Rubinstein, J. L., M. L. Rocca, J. E. Vidale, K. C. Creager, and A. G. Wech (2008), Tidal modulation of nonvolcanic tremor, Science, 319(5860), 186-189.

Schwartz, S. Y., and J. M. Rokosky (2007), Slow slip events and seismic tremor at circum-Pacific subduction zones, Rev. Geophys., 45, RG3004, doi:10.1029/2006RG000208.

Segall, P. (2010), Earthquake and Volcano Deformation, Princeton Univ. Press, Princeton, N. J.

Shelly, D. R., Z. Peng, D. P. Hill, and C. Aiken (2011), Triggered creep as a possible mechanism for delayed dynamic triggering of tremor and earthquakes, Nat. Geosci., 4, 384-388.

Shibazaki, B., and Y. Iio (2003), On the physical mechanism of silent slip events along the deeper part of the seismogenic zone, Geophys. Res. Lett., 30(9), 1489, doi:10.1029/2003GL017047.

Shibazaki, B., and T. Shimamoto (2007), Modelling of short-interval silent slip events in deeper subduction interfaces considering the frictional properties at the unstable-stable transition regime, Geophys. J. Int., 171, 191-205.

Shimamoto, T. (1986), Transition between frictional slip and ductile flow for halite shear zones at room temperature, Science, 231, 711-714.

Thomas, A. M., R. M. Nadeau, and R. Bürgmann (2009), Tremor-tide correlations and near-lithostatic pore pressure on the deep San Andreas fault, Nature, 462, 1048-1051.

Thomas, A. M., R. Bürgmann, D. R. Shelly, N. M. Beeler, and M. L. Rudolph (2012), Tidal triggering of low frequency earthquakes near Parkfield, California: Implications for fault mechanics within the brittle-ductile transition, J. Geophys. Res., 117, B05301, doi:10.1029/2011JB009036. 